\title{
Artigo/Article
}

\section{Estudo clínico-radiológico de crianças e adolescentes indígenas Suruí, Região Amazônica}

\author{
Clinical and radiological study of Suruí indigenous children and adolescents, Amazon Region, Brazil
}

\author{
Paulo Cesar Basta ${ }^{1}$, Diana Patrícia Giraldo Rios ${ }^{1}$, Luiz Carlos Corrêa Alves ${ }^{1}$, Clemax Couto Sant' Anna \\ e Carlos Everaldo Alvares Coimbra Junior ${ }^{1}$
}

\begin{abstract}
RESUMO
Introdução: Entre os Suruí de Rondônia foram registradas incidências médias de TB > 2.500/100.000 habitantes, entre 1991-2002. Aproximadamente 50\% desses casos foram notificados em < 15 anos. Métodos: Trata-se de um estudo clínico-epidemiológico que teve como objetivo descrever as características clínico-radiológicas em crianças e adolescentes identificados como contatos de doentes de TB. Além disto, aplicar o sistema de pontuação para o diagnóstico de TB na infância e verificar se as condutas adotadas no nível local foram concordantes com as diretrizes nacionais. Resultados: Foram analisados 52 Rx de 37 indígenas. Deste conjunto, 48,1\% foram normais e 51,9\% anormais. Alguns dos Rx apresentaram duas ou mais alterações, totalizando 36 eventos independentes. Observou-se infiltrados (38,9\%), calcificações $(38,9 \%)$, cavitações (11,1\%) e atelectasias/derrame pleural ( $11,1 \%)$. Nas imagens anormais, $22,2 \%$ eram TB provavelmente ativa e $33,3 \%$ sequelas. A confrontação com as diretrizes constatou $52,6 \%$ de condutas discordantes. Conclusões: A presença da infecção tuberculosa latente (ITBL) e TB ativa, entre crianças e adolescentes, são indicadores de transmissão ativa e continuada do Mycobacterium tuberculosis. Os Rx mostrando alta frequência de infiltrados e calcificações é compatível com primo-infecção em idade precoce. Entretanto, essas alterações não são diferentes daquelas observadas entre outros grupos, não sugerindo comprometimento imunológico. As discordâncias apontadas indicam que o momento ideal para o tratamento da ITBL passou despercebido. Conclui-se que é fundamental a utilização do sistema de pontuação para o correto diagnóstico de TB na infância, assim como a realização de baciloscopia e cultura de escarro em adolescentes capazes de expectorar.
\end{abstract}

Palavras-chaves: Tuberculose na infância. Sistema de pontuação para diagnóstico. Achados radiológicos. Índios sulamericanos. Saúde indígena.

\begin{abstract}
Introduction: The average incidence coefficient of tuberculosis in Suruí Indians from Rondônia was $2.500 / 100.000$ inhabitants in 1991-2002. About 50\% of these cases were reported in children $<15$ years-old. Methods: This study aimed to describe the clinical and radiological characteristics of children and adolescents identified as TB case contacts. A score system for the diagnosis of childhood TB was used and the procedures adopted by local health services were in accordance with national guidelines. Results: 52 chest X-rays of 37 indigenous subjects were analyzed; of these, $51.9 \%$ were abnormal. Some X-rays showed more than two lesions, making a total of 36 independent events. Infiltrates (38.9\%), calcifications (38.9\%), cavitations $(11.1 \%)$ and atelectasis/pleural effusion (11.1\%) were observed. Among the abnormal images, $22.2 \%$ were probably indicated active TB and $33.3 \%$ showed sequelae. Confrontation with the guidelines revealed 52.6\% of divergent procedures. Conclusions: The presence of latent tuberculosis infection (LTBI) and active TB between children and adolescents are indicators of active and progressive transmission of Mycobacterium tuberculosis. The X-rays showed high frequencies of infiltrates and calcifications, which are compatible with primary infection in early childhood. However, these lesions are not different from those observed among other groups and do not suggest immune deficiencies. The divergences presented show that the best moment for the treatment of LTBI went unnoticed by local personnel. In conclusion, the use of a score system is fundamental for the correct diagnosis of TB in childhood, as is conducting bacilloscopy and sputum culture in adolescents able to expectorate.
\end{abstract}

Key-words: Tuberculosis in childhood. Score system for diagnosis. Radiological findings. South American Indians. Indigenous health.

1. Escola Nacional de Saúde Pública Sergio Arouca, Fundação Oswaldo Cruz, Rio de Janeiro, RJ. 2. Instituto de Puericultura e Pediatria Martagão Gesteira, Universidade Federal do Rio de Janeiro. Rio de Janeiro, RJ.

Endereço para correspondência: Dr. Paulo Cesar Basta. ENSP/FIOCRUZ. R. Leopoldo Bulhões 1480, 21041-210 Rio de Janeiro, RJ.

Tel: 5521 2598-2683

e-mail: paulobasta@gmail.com

Recebido para publicação em 26/03/2010

Aceito em 20/08/2010

\section{INTRODUÇÃO}

NoBrasil, estima-se que cerca de 80.000 casos novos detuberculose (TB) são diagnosticados ao ano, dos quais 23.520 afetam crianças e adolescentes menores de 15 anos. Isto representa incidência média de 66 casos por 100.000 habitantes para esse grupo etário ${ }^{1,2}$.

Entre os povos indígenas no Brasil, a TB constitui importante endemia, que se expressa sobremaneira sobre o contingente populacional menor de 15 anos de idade ${ }^{3-5}$.

O povo Suruí de Rondônia situa-se entre os grupos indígenas do país nos quais se verificam as maiores incidências médias anuais de TB, sendo que no período 1991 a 2002 ultrapassaram-se os 2.500 casos por 100.000 habitantes $^{6}$. Nesse período, aproximadamente $50 \%$ dos casos foram notificados em crianças menores de 15 anos. Merece destaque também o fato que somente $43,2 \%$ de todas as notificações no período terem sido confirmadas pelo exame direto do escarro. Além disto, não houve registro da realização de outros procedimentos, tais como teste tuberculínico, exame histopatológico e cultura, para se firmar o diagnóstico de TB entre os casos indígenas analisados ${ }^{6}$.

Embora o número anual de casos notificados seja expressivo, a situação epidemiológica da TB entre crianças e adolescentes no Brasil é pouco conhecida, especialmente entre indígenas. A intenção deste artigo é, de forma inédita no país, propor o diagnóstico de TB em crianças e adolescentes indígenas a partir da utilização de um protocolo nacional validado por Sant'Anna cols ${ }^{7}$, Maciel cols ${ }^{8}$ e Pedrozo cols ${ }^{9}$ com crianças e adolescentes não-indígenas. Desta forma, pretende-se contribuir para ilustrar as principais dificuldades envolvidas na identificação da doença nesse grupo etário particularmente vulnerável da população.

Nesse sentido, o objetivo desse estudo é descrever as características clínico-radiológicas e aplicar o sistema de pontuação para diagnóstico da TB na infância em crianças e adolescentes indígenas Suruí, contatos de doentes de TB. Além disto, verificar se as condutas adotadas pelo serviço local de saúde foram concordantes com as diretrizes nacionais para diagnóstico de TB na infância ${ }^{10}$. 


\section{MÉTODOS}

Esta investigação insere-se em um projeto de pesquisa ampliado e iniciado em 2003, denominado Tuberculose em populações indígenas da Amazônia: uma abordagem interdisciplinar entre os Suruí de Rondônia.

O povo indígena Suruí localiza-se na Terra Indígena Sete de Setembro, situada na divisa entre os Estados de Rondônia e Mato Grosso $\left(60-61^{\circ} \mathrm{W}, 10-11^{\circ} \mathrm{S}\right)$. À época da pesquisa de campo, realizada entre fevereiro e maio de 2005, a população encontrava-se distribuída em 12 aldeias, e totalizava 993 pessoas, das quais $64,2 \%$ eram crianças e adolescentes de 0 a $19 \operatorname{anos}^{11}$.

Em 2005, foi realizado um inquérito tuberculínico com PPD Rt 23 em 645 indígenas Suruí, dos quais 430 (66,7\%) eram crianças e adolescentes menores de 19 anos. Entre esses Suruí menores de 19 anos, testados com PPD, 69 (16\%) apresentaram reações $\geq 10 \mathrm{~mm}$, dos quais $32(46,4 \%)$ já haviam realizado tratamento anterior para $\mathrm{TB}^{11}$. Portanto, naquele contexto foi indicado o tratamento da infecção tuberculosa latente (ITBL) para 37 crianças e adolescentes que eram contatos de doentes de TB, porque apesar delas se encontrarem assintomáticas (ausência de tosse, febre e/ou emagrecimento), o teste tuberculínico revelou-se $\geq 10 \mathrm{~mm}$.

Em 2007, com auxílio das equipes multidisciplinares de saúde do Distrito Sanitário Especial Indígena de Vilhena foi possível localizar $19(51,4 \%)$ crianças e adolescentes do total de 37 para as quais havia sido indicado o tratamento de ITBL. Foi realizado estudo clínicoepidemiológico pormenorizado nessas 19 crianças e adolescentes, das quais foram coletados dados relativos à data de nascimento, aldeia de residência, sinais e sintomas de $\mathrm{TB}$, situação vacinal com BCG e exames radiológicos anteriores. Foram efetuadas ainda medidas antropométricas (peso e estatura), novo teste tuberculínico e nova radiografia de tórax. Também, se aplicou o sistema de pontuação para diagnóstico de TB na infância contido na II Diretrizes Brasileiras para o Controle da Tuberculose ${ }^{10}$. As radiografias foram avaliadas conjuntamente por três dos autores (Alves, Rios e Sant'Anna), de acordo com os critérios propostos por Boon cols ${ }^{12}$.

Os dados foram armazenados em planilhas eletrônicas e as análises descritivas foram efetuadas com auxílio do pacote Statistical Package for the Social Science - SPSS (versão 9.0).

\section{Ética}

Este projeto foi aprovado pelo Comitê de Ética em Pesquisa da Escola Nacional e Saúde Pública Sergio Arouca (CEP/ENSP) e pela Comissão Nacional de Ética em Pesquisa (CONEP) do Conselho Nacional de Saúde.

\section{RESULTADOS}

Foi possível recuperar as radiografias das 37 crianças e adolescentes indígenas Suruí indicadas ao tratamento da ITBL em 2005. Destas crianças, cinco realizaram 2 exames radiológicos, cinco realizaram 3 exames e 27 realizaram uma única radiografia, em diferentes momentos, totalizando 52 imagens para o estudo das alterações radiológicas.

O estudo radiológico revelou que $25(48,1 \%)$ radiografias foram classificadas como normais e 27 (51,9\%) como anormais. A Tabela 1 mostra que parte das radiografias anormais apresentou duas ou mais alterações, totalizando 36 eventos independentes para análise, distribuídos da seguinte maneira: infiltrados em 38,9\% (14/36), calcificações em 38,9\% (14/36), cavitações em 11,1\% (4/36) e atelectasias e/ou derrame pleural em 11,1\% (4/36).

As regiões pulmonares mais acometidas foram os terços superiores. Dentre as 27 imagens anormais, $6(22,2 \%)$ foram consideradas como TB, provavelmente ativa, e $9(33,3 \%)$ como sequelas. As alterações restantes $(44,5 \%)$ foram consideradas sugestivas de outras doenças.

Entre as 19 crianças e adolescentes, que foram avaliados individualmente, cinco haviam recebido tratamento para TB pulmonar com esquema I (RHZ) e seis haviam realizado o tratamento da infecção tuberculosa latente apenas com isoniazida $(\mathrm{H})$.

A média de idade das 19 crianças e adolescentes analisados foi de 11,3 anos (variando de 3,5 a 16,4 anos), com mediana de 12,6 anos. Dentre as 11 crianças que receberam tratamento para TB pulmonar ou para a ITBL, observou-se que $9(81,8 \%)$ eram do sexo feminino.

A análise do indicador peso por idade revelou que três crianças com 6, 8 e 10 anos de idade, respectivamente, encontravam-se abaixo do percentil 10, enquanto um adolescente de 15 anos apresentava índice de massa corporal (IMC) abaixo de $18 \mathrm{~kg} / \mathrm{m}^{2}$, totalizando 21,1\% da amostra com déficit nutricional (Tabela 2).

Quanto à situação vacinal, as 19 crianças e adolescentes, avaliados individualmente, haviam sido revacinadas com BCG intradérmico em $2005^{11}$; portanto, apresentavam pelo menos uma cicatriz vacinal.

Foram comparadas as condutas adotadas pelo serviço local de saúde em Rondônia com as preconizadas pelas II Diretrizes para o Controle da TB e constatou-se discordância em 52,6\% (10/19) dos procedimentos indicados (Tabela 2 ).

\begin{tabular}{|c|c|c|}
\hline Alteração radiológica predominante & Número & Percentagem \\
\hline Infiltrados & 14 & 38,9 \\
\hline Calcificações & 14 & 38,9 \\
\hline Cavitações & 4 & 11,1 \\
\hline Atelectasias e/ou derrame pleural & 4 & 11,1 \\
\hline Total & 36 & 100,0 \\
\hline
\end{tabular}


TABELA 2 - Dados clínico-radiológicos de crianças e adolescentes indígenas Suruí (RO), contatos de TB, agosto 2007.

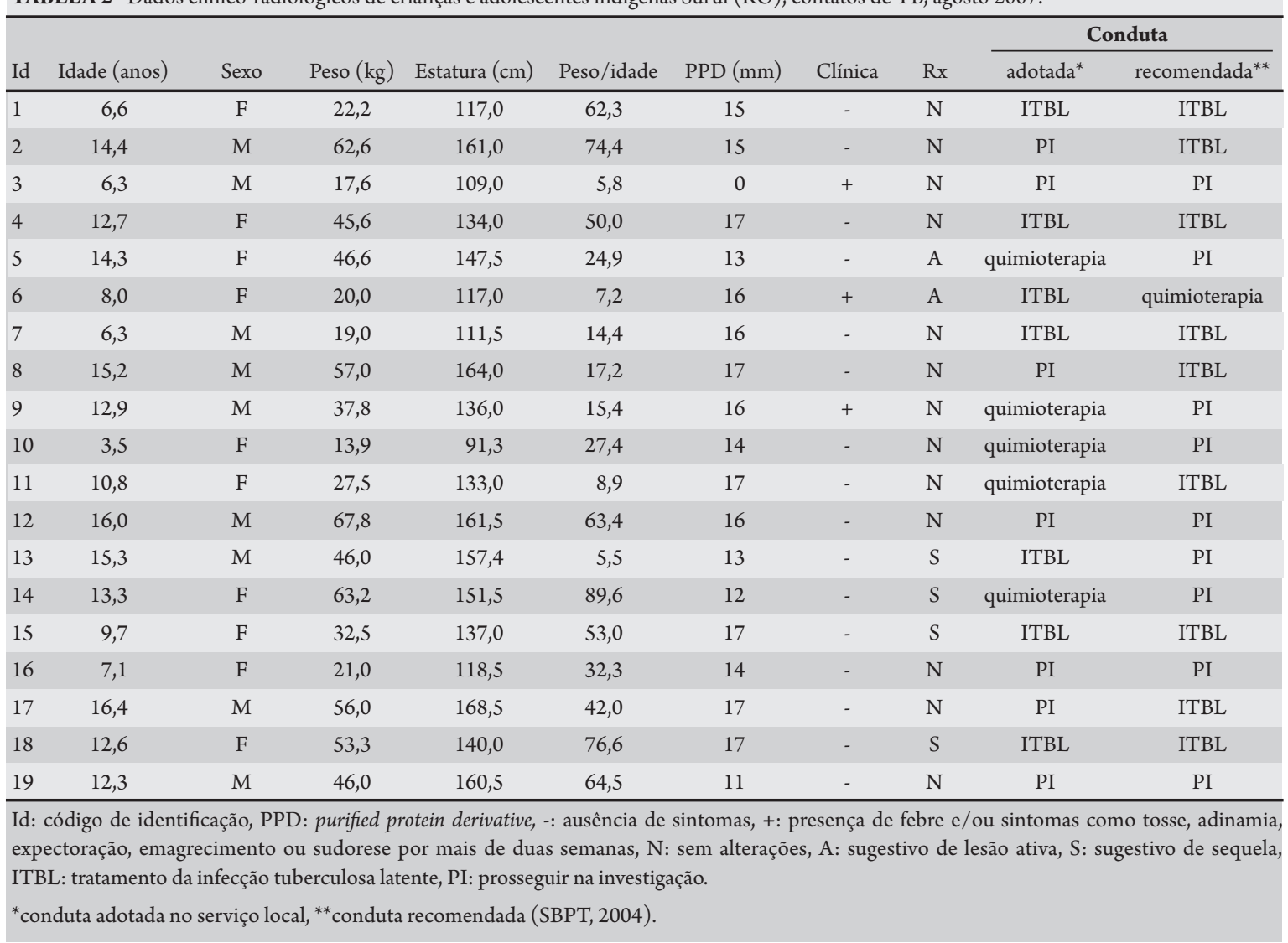

\section{DISCUSSÃO}

A análise do conjunto de dados provenientes das imagens radiológicas $\left(\mathrm{n}^{\mathrm{o}}=52\right)$ de 37 pessoas, somada à avaliação clínica individual de 19 crianças e adolescentes, revelam que a TB, de fato, constitui-se como relevante problema de saúde pública entre os Suruí de Rondônia. A presença da infecção tuberculosa latente e a doença em atividade, entre crianças e adolescentes, são indicadoras de transmissão ativa e continuada de Mycobacterium tuberculosis na população.

A análise das radiografias, mostrando alta frequência de infiltrados e calcificações, é compatível com a ocorrência de primo-infecção em idade precoce. No entanto, as alterações radiológicas encontradas não são diferentes daquelas observadas entre crianças e adolescentes de outros grupos populacionais ${ }^{13-17}$. Além disto, a quase totalidade dos casos avaliados individualmente (18/19) apresentaram reações ao teste tuberculínico de grande intensidade (variação de 11 a $17 \mathrm{~mm}$ ). Portanto, não há motivo para se supor que possa haver algum comprometimento imunológico dos indígenas analisados.

Em contrapartida, as precárias condições sócio-econômicas observadas nas aldeias, onde as habitações costumam ser mal ventiladas, pouco iluminadas e com muitos habitantes em dormitórios únicos, associada a presença de crianças com déficit nutricional constituem-se como fatores que podem favorecer a transmissão da TB, contribuindo desta forma para a manutenção da endemia na comunidade.

O problema da TB na infância é mais evidente entre contatos de adultos bacilíferos, e seu diagnóstico requer abordagem integrada entre dados clínicos, radiológicos e epidemiológicos, pois o isolamento do Mycobacterium tuberculosis no escarro é difícil, nesta faixa etária ${ }^{18}$.

Enquanto a baciloscopia de escarro é capaz de identificar cerca de $75 \%$ dos casos de TB pulmonar entre adultos, menos de $20 \%$ das crianças diagnosticadas com TB apresentam resultados positivos ao exame direto do escarro. Mesmo quando se obtém boas amostras de escarro, provenientes de lavado gástrico, a positividade à cultura não ultrapassa $50 \%{ }^{19}$. Além disto, neste grupo etário os sintomas se confundem com outras doenças e os achados radiológicos são poucos específicos, o que traz dificuldades adicionais para o correto diagnóstico ${ }^{20,21}$.

Com a intenção de enfrentar esse problema, algoritmos baseados num conjunto de dados clínicos e epidemiológicos têm sido desenvolvidos em várias partes do mundo para reduzir os erros diagnósticos entre crianças e adolescentes ${ }^{22-27}$. No Brasil, o Ministério da Saúde recomenda o uso de um sistema de pontuação para o diagnóstico de TB na infância desde $2002^{28}$. Esse sistema foi validado em diferentes momentos e mostrou sensibilidade de $86 \%$ e especificidade entre $87 \%$ e $100 \%$.

Para tanto, nas diretrizes brasileiras é recomendado que toda criança ou adolescente com suspeita clínica de TB seja avaliada por meio desse sistema de pontuação que pressupõe a análise combinada de achados clínico-radiológicos, a interpretação do teste tuberculínico, a observância da situação vacinal com BCG e do estado nutricional, e a história de contato com adulto doente de tuberculose. Quando a pontuação for igual ou superior a 30, o diagnóstico é possível e o tratamento para TB deve ser considerado. Se a pontuação for inferior a 30 o diagnóstico é pouco provável, e deve-se prosseguir na investigação do caso ${ }^{10}$. 
A aplicação do sistema de pontuação para o diagnóstico de TB na infância revelou discordâncias com os diagnósticos, eminentemente clínicos, adotados pelo serviço local de saúde. As condutas foram discordantes nos seguintes aspectos: a) cinco tratamentos foram iniciados sem que houvesse elementos suficientes para firmar o diagnóstico de TB; b) três casos em que se deveria realizar o tratamento para ITBL não foi realizado qualquer procedimento; c) um doente que recebeu tratamento para ITBL, deveria ter realizado a quimioterapia para $\mathrm{TB}$; d) um caso no qual foi realizado o tratamento para ITBL, deveria ter sido realizada investigação complementar para se adotar uma conduta mais adequada.

As discordâncias apontadas acima permitem supor que o momento ideal para se realizar o tratamento da ITBL, entre as crianças e adolescentes Suruí tenha passado despercebido. Este fato é revestido de adicional preocupação, pois em crianças abaixo de 5 anos de idade não vacinadas com BCG e portadoras de ITBL, provenientes de países com média incidência de TB, como o Brasil, o risco de desenvolver TB ativa pode ultrapassar os $40 \%^{19}$.

Conclui-se que para um correto diagnóstico e conhecimento mais preciso sobre a epidemiologia da TB entre crianças e adolescentes é de fundamental importância a utilização do sistema de pontuação para o diagnóstico de TB na infância, assim como a realização de baciloscopia e cultura de escarro em adolescentes capazes de expectorar ${ }^{13}$.

Além disto, o exame precoce dos contatos deve ser incorporado à rotina de vigilância e controle da TB entre os grupos indígenas no país por meio do uso sistematizado e simultâneo da radiografia de tórax e do teste tuberculínico. Naqueles casos em que for diagnosticada infecção tuberculosa latente, o tratamento supervisionado com isoniazida deve ser iniciado prontamente.

Em nossa opinião, somente desta maneira é que se vislumbrará uma redução do impacto da endemia sobre esse segmento da população.

\section{CONFLITO DE INTERESSE}

Os autores declaram não haver nenhum tipo de conflito de interesse no desenvolvimento do estudo.

\section{SUPORTE FINANCEIRO}

Conselho Nacional de Desenvolvimento Científico e Tecnológico Processo 47.0850/2004-3.

\section{REFERÊNCIAS}

1. World Health Organization [Internet]. Global tuberculosis control-surveillance, planning, financing. 2007 - [acesso em 06/02/2010]. Disponível em: www.who. int/tb/publications/global_report/2007/pdf/full.pdf

2. Nelson LJ, Wells CD. Global epidemiology of childhood tuberculosis. Int J Tuberc Lung Dis 2004; 8:636-647.

3. Coimbra-Jr CEA, Basta PC. The burden of tuberculosis in indigenous peoples in Amazonia, Brazil. Trans R Soc Trop Med Hyg 2007; 101:635-636.

4. Garnelo L, Brandão LC, Levino A. Dimensões e potencialidades dos sistemas de informação geográfica na saúde indígena. Rev Saude Publica 2005; 39:634-640

5. Escobar AL, Coimbra-Jr CEA, Camacho LAB, Portela MC. Tuberculose em populações indígenas de Rondônia, Amazônia, Brasil. Cad Saude Publica $2001 ; 17: 285-298$
6. Basta PC, Coimbra-Jr CEA, Escobar AL, Santos RV. Aspectos epidemiológicos da tuberculose na população indígena Suruí, Amazônia, Brasil. Rev Soc Bras Med Trop 2004; 37:338-342.

7. Sant'Anna CC, Orfaliais CTS, March MF. A restrospective evaluation of a score system adopted by the Ministry of Health, Brazil in diagnosis of pulmonary tuberculosis in childhood: a case control study. Rev Inst Med Trop Sao Paulo 2003; 45:103-105.

8. Maciel ELN, Dietze R, Silva REC, Hadad DJ, Struchiner C. Avaliação de sistemas de pontuação para o diagnóstico de tuberculose na infância preconizado pelo Ministério da Saúde, Brasil. Cad Saude Publica 2008; 24:402-408.

9. Pedrozo C, Sant'Anna C, March MF, Lucena S. Clinical scoring system for paediatric tuberculosis in HIV-infected and non-infected children in Rio de Janeiro. Int J Tuberc Lung Dis 2009; 13:413-415.

10. Sociedade Brasileira de Pneumologia e Tisiologia. II Consenso Brasileiro de Tuberculose: Diretrizes Brasileiras para Tuberculose. J Bras Pneumol 2004; 30 (supl 1):S1-S55.

11. Basta PC, Coimbra-Jr CEA, Camacho LAB, Santos RV. Risk of tuberculous infection in an indigenous population from Amazonia, Brazil. Int J Tuberc Lung Dis 2006b; 10:1354-1359.

12. Boon SD, Bateman ED, Enarson DA, Borgdorff MW, Verner S, Lombard CJ, et al. Development and evaluation of a new chest radiograph reading and recording system for epidemiological surveys of tuberculosis and lung disease. Int J Tuberc Lung Dis 2005; 9:1088-1096.

13. Sant'Anna C, March MF, Barreto M, PereiraS, Schmidt C. Pulmonary tuberculosis in adolescents: radiographic features. Int J Tuberc Lung Dis 2009; 13:1566-1568

14. Burfill J, Williams CJ, Brain G, Conder G, Hine AL, Misra RR. Tuberculosis: a radiologic review. Radiographics 2007; 27:1255-1273.

15. American Thoracic Society. Diagnostic standards and classification of tuberculosis in adults and children. Am J Respir Crit Care Med 2000; 161:1376-1395.

16. Leung NA. Pulmonary tuberculosis: The essentials. Radiology 1999; 210: 307-322.

17. McAdams HP, Erasmus J, Winter JA. Radiologic manifestations of pulmonary tuberculosis. Radiol Clin North Am 1995; 33:655-678.

18. Sant'Anna, CC. Tuberculose na infância e na adolescência. Rio de Janeiro: Editora Atheneu; 2002.

19. Starke JR. Childhood tuberculosis. A diagnostic dilemma. Chest 1993; 104: 329-330.

20. Sant'Anna CC, Hijjar MA. Recent contribution of the World Health Organization to control childhood tuberculosis. Rev Saude Publica 2007; 41:117-120.

21. Sant'Anna CC, Orfaliais CT, March MF, Conde MB. Evaluation of a proposed diagnostic score system for pulmonary tuberculosis in Brazilian children. Int J Tuberc Lung Dis 2006; 10:463-465.

22. Stegen G, Jones K, Kaplan P. Criteria for guidance in the diagnosis of tuberculosis. Pediatrics 1969; 49:260-263.

23. Nair PM, Philip E. A Scoring system for the diagnosis of tuberculosis in children. Indian Pediatr 1981; 18:299-303.

24. Ghidey Y, Habte D. Tuberculosis in childhood: an analysis of 412 cases. Ethiop Med J 1983; 21:161-167.

25. Tidjani O, Amedone A, Ten Dam HG. The protective effect of BCG vaccination of the newborn aginst childhood tuberculosis in Africa community. Tubercle 1986; 67:269-281.

26. Crofton J, Horne N, Miller F. Clinical tuberculosis. London: Macmillan Education; 1992.

27. Fourie PB, Becker PJ, Festenstein F, Migliori GB, Alcaide J, Antunes M, et al. Procedures for developing a simple scoring method based on unsophisticated criteria for screening children for tuberculosis. Int J Tuberc Lung Dis 1998; 2:116-123.

28. Fundação Nacional de Saúde. Tuberculose: Guia de Vigilância Epidemiológica. Brasília: Ministério da Saúde; 2002. 